\title{
Síndrome hemofagocítico en un trasplantado renal con síndrome de Alport
}

\author{
JORGE VEGA ${ }^{1,2}$, MARÍA DE LOS ÁNGELES RODRÍGUEZ ${ }^{1,3}$, \\ HELMUTH GOECKE ${ }^{1,2}$, MARIO SANTAMARINA ${ }^{4}$
}

\section{Hemophagocytic syndrome after kidney transplant in a patient with hereditary nephritis. Report of one case}

We report a 28-year-old male with a hereditary nephritis (Alport Syndrome) on hemodialysis for 5 years, who received a kidney graft from a deceased donor. Cyclosporine (CsA), mycophenolate mofetil (MMF) and steroids were prescribed. In the postoperative period the patient had thrombophlebitis and diarrhea. A CT scan showed splenomegaly, ascites, bilateral pleural effusion and bowel edema. Laboratory showed hypoalbuminemia, increased $C$ reactive protein (CRP) and panhypogammaglobulinemia. At day 32 after transplantation, an acute rejection (Banff II b) was diagnosed and treated with methylprednisolone, replacing CsA by tacrolimus. The acute rejection was controlled but six days later, high fever, pancytopenia and hyperferritinemia appeared. A bone marrow smear showed numerous histiocytes and hemophagocytosis. Hemophagocytic syndrome was diagnosed. MMF and tacrolimus were withdrawn and CsA was reinstituted. Fever fell quickly, CPR normalized at 24 hours and white blood cell count at 72 hours. Days later, the concentrations of albumin, immunoglobulins and hematological parameters normalized. The patient was discharged on day 57 after admission in good condition.

(Rev Med Chile 2013; 141: 519-524).

Key words: Lymphohistiocytosis, hemophagocytic; Mycophenolate mofetil; Nephritis, hereditary; Organ transplantation.

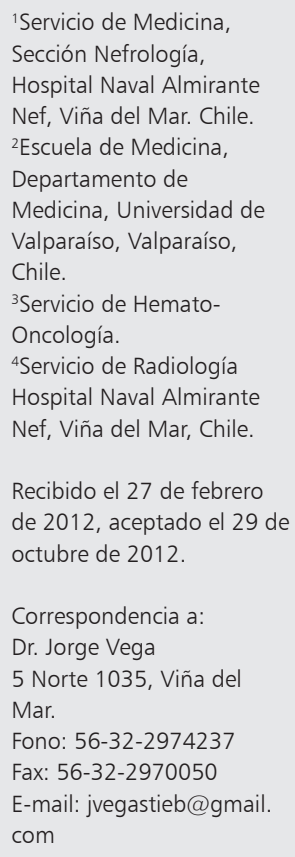

E 1 síndrome hemofagocítico (SHF), linfohistiocitosis hemofagocítica o síndrome de activación macrofágica es un trastorno raro, caracterizado por activación y proliferación excesiva no neoplásica de macrófagos ${ }^{1,2}$. Un hallazgo característico es la eritrofagocitosis por histiocitos $^{3}$. Existe una forma familiar, que es transmitida genéticamente y una reactiva a infecciones, neoplasias malignas, enfermedades autoinmunes y trasplantes ${ }^{4-6}$. El SHF tiene una elevada mortalidad $^{4-7}$. Comunicamos un caso de SHF ocurrido durante el postoperatorio de un trasplante renal.

\section{Caso clínico}

Hombre de 28 años, portador de un síndrome de Alport que después de 5 años en hemodiálisis recibió un trasplante renal $(\mathrm{TxR})$ de donante fallecido.

Al cuarto año en hemodiálisis presentó pancitopenia. El mielograma mostró dishemopoyesis con caracteres sugerentes de mielodisplasia, el cariotipo en médula ósea fue normal. Se presumió una etiología autoinmune y se indicó ciclosporina (CsA). Al mes, los leucocitos habían subido desde 2.700 a $4.000 \mathrm{~mm}^{3}$, las plaquetas de 126.000 a $160.000 \mathrm{~mm}^{3}$ y el hematocrito de 27 a $36,5 \%$. Continuó recibiendo CsA hasta el TxR, 15 meses después.

Previo al trasplante, dado que no sería prudente utilizar azatioprina por su efecto mielodepresor, se ensayó el uso de micofenolato mofetil (MMF) en dosis progresivas hasta $2.000 \mathrm{mg} /$ día, para conocer su tolerancia medular. No se originaron 
citopenias durante 6 semanas de uso, decidiéndose que podría emplearse en un TxR futuro.

El día del TxR se prescribió CsA, MMF y corticoides. Si bien el injerto produjo orina de inmediato, la depuración fue escasa, por lo que se dializó en 3 ocasiones. El día 8 se diagnosticó una tromboflebitis iliofemoral derecha, iniciándose terapia anticoagulante. El día 12 tuvo diarrea, vómitos y dolores cólicos, lo que motivó reducir la dosis de MMF y prescribir metronidazol y ciprofloxacina, por la eventualidad de una etiología infecciosa. Los coprocultivos, coproparasitológicos, toxina de Clostridium difficile y rotavirus fueron negativos. La diarrea cedió completamente 7 días después. El día 26 presentó cefalea y un nuevo episodio de diarrea, vómitos y dolor abdominal. No tuvo fiebre. El scanner mostró ligera esplenomegalia, engrosamiento de la pared intestinal, derrame pleural, ascitis abundante y un linfocele en la cara anterior del riñón trasplantado, al cual desplazaba hacia dorsal (Figura 1). De los exámenes destacó elevación de la proteína $\mathrm{C}$ reactiva (PCR) a 18,7 $\mathrm{mg} / \mathrm{L}(\mathrm{VN}:<1)$ e hipoalbuminemia severa $(1,8 \mathrm{~g} /$ dl). Se prescribió metronidazol y ceftriaxona. No se demostró infección y la diarrea cedió en 5 días.

El día 32 presentó bruscamente un dolor intenso en el área del injerto con ascenso de la creatinina y uremia, acidosis metabólica y reducción del volumen urinario. Un scanner (solicitado por sospecha de ruptura renal) mostró ascitis de mayor cuantía, engrosamiento de la pared del colon y aumento del tamaño del linfocele, manteniéndose las alteraciones del scanner anterior. El riñón trasplantado no presentaba ruptura. Se diagnosticó un rechazo agudo y se trató con metilprednisolona 1 gramo por 3 días, sustituyendo la CsA por tacrolimus. La azotemia descendió hasta estabilizarse la creatinina en $2,4 \mathrm{mg} / \mathrm{dl}$. Se puncionó el linfocele obteniendo $725 \mathrm{ml}$.

El día 38 apareció fiebre $>38^{\circ} \mathrm{C}$ sin causa aparente, asociado a una rápida caída del hematocrito $(34,5$ a $23,8 \%)$, leucopenia $\left(3.000 \mathrm{~mm}^{3}\right)$, trombocitopenia $\left(130.000 \mathrm{~mm}^{3}\right)$ y elevación de la PCR. Un test de Coombs directo fue negativo. Se suspendió el MMF por sospecha de depresión medular. En los días siguientes continuó anemizándose (Hto 20,7\%), se acentuó la leucopenia $\left(2.700 \mathrm{~mm}^{3}\right)$, persistió la hipoalbuminemia $(1,8$ g/dl), apareció una panhipogammaglobulinemia severa y se constató una baja de peso de 4 kilos.

El linfocele comenzó a deformar la pared

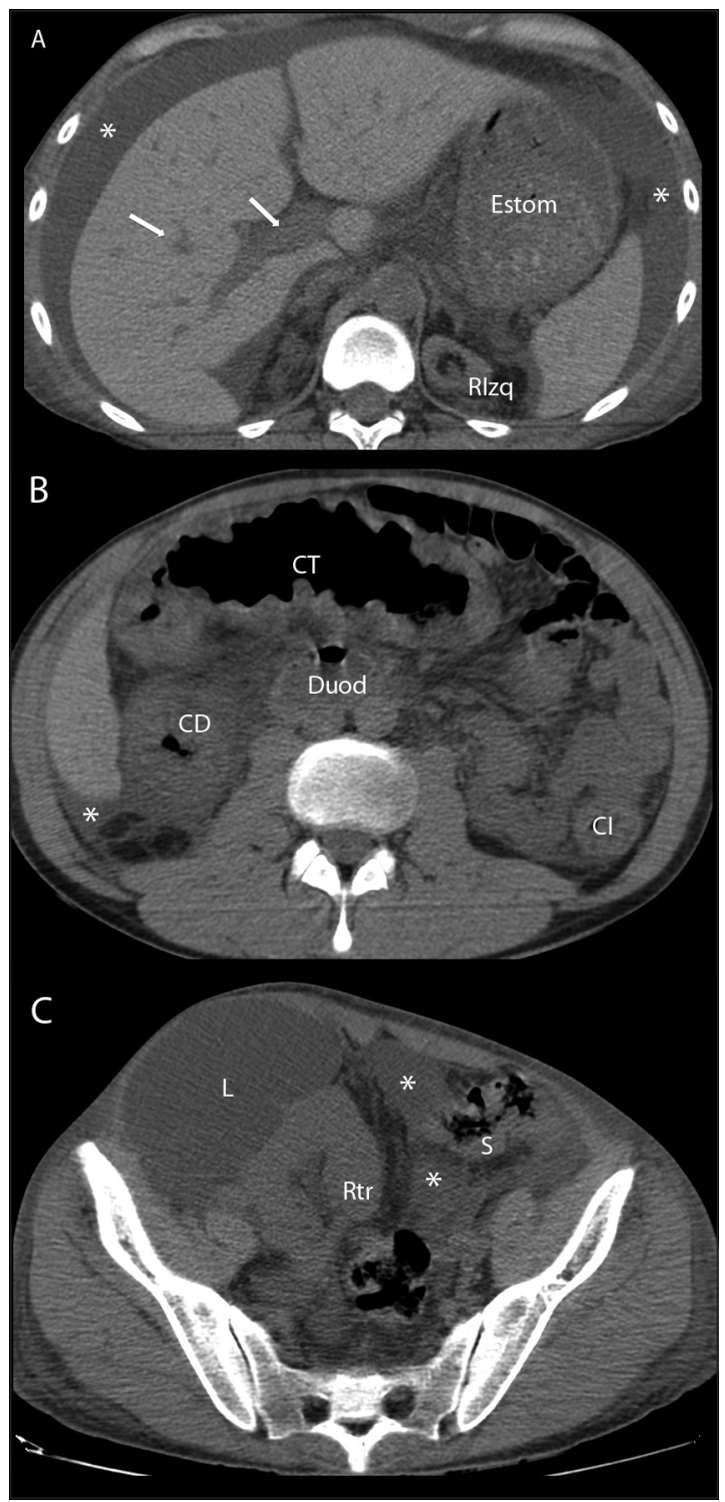

Figura 1. TC de abdomen/pelvis sin contraste EV. A. Imagen axial a nivel del abdomen superior, donde se evidencia la presencia de líquido ascítico en recesos superiores de la cavidad peritoneal $\left(^{*}\right)$. Se observa una disminución de la atenuación de las estructuras vasculares hepáticas (flechas blancas), que aumentan el contraste en relación al parénquima hepático, hallazgo relacionado con una disminución del hematocrito. RIzq: riñón izquierdo nativo atrófico. Estom: Estómago. B. En un corte más caudal, se aprecia un marcado engrosamiento de la pared del colon (CD: colon derecho; CT: colon transverso; $\mathrm{Cl}$ : colon izquierdo; Duod: duodeno). Presencia de ascitis (*). C. A nivel pelviano, el riñón trasplantado (Rtr) en relación a su superficie anterolateral, se encuentra parcialmente comprimido por un linfocele (L). Presencia de ascitis (*). S: colon sigmoides. 
abdominal efectuándose su marsupialización el día 43. La persistencia de la anemia y leucopenia hizo plantear una enfermedad linfoproliferativa post-trasplante (ELPPT) o recurrencia de su mielodisplasia. El mielograma y citometría de flujo descartaron una ELPPT. En la médula ósea se observó abundantes histiocitos y hemofagocitosis (Figura 2). Con diagnóstico presuntivo de un SHF-asociado a trasplante se mantuvo suspendido el MMF y se cambió el tacrolimus por CsA, manteniendo la prednisona en $10 \mathrm{mg} /$ día. Las concentraciones de ferritina y triglicéridos estaban elevadas ( $1.873 \mathrm{ng} / \mathrm{ml}$ y $228 \mathrm{mg} / \mathrm{dl}$, respectivamente) y la de fibrinógeno normal (360 mg/dl). La PCR se normalizó a las $24 \mathrm{~h}$ de iniciada la CsA y el recuento de leucocitos a las $72 \mathrm{~h}$. En los días siguientes el estado general mejoró progresivamente, la fiebre cedió y el hematocrito fue ascendiendo paulatinamente, pero la función renal se mantuvo estacionaria (creatinina 2,6 mg/dl). La biopsia renal mostró un rechazo agudo BANFF II b, arterioloesclerosis y una lesión glomerular proliferativa; que se atribuyó al donante. Los anticuerpos antinucleares, ANCA y las concentraciones de C'3 y C'4 fueron normales.

Al día 56 , la creatininemia fue $2,7 \mathrm{mg} / \mathrm{dl}$ y no habían signos de inflamación y la cuantificación de inmunoglobulinas IgG, IgA e IgM y la albuminemia eran normales. El paciente fue dado de alta.

En el control ambulatorio cuatro semanas después del egreso sus parámetros hematológicos se habían normalizado y la función renal era mejor (creatinina $2,4 \mathrm{mg} / \mathrm{dl}$, clearance de creatinina 33 $\left.\mathrm{ml} / \mathrm{min} / 1,73 \mathrm{~m}^{2}\right)$.

\section{Discusión}

El SHF es un síndrome inflamatorio que puede ser indistinguible de una sepsis, falla orgánica múltiple (FOM) o acompañarse de ellas ${ }^{3,8,9}$.

En el SHF hay una desregulación de la función de los linfocitos Th-1 con activación y proliferación de linfocitos $\mathrm{T}$ e histiocitos, los que infiltran órganos y secretan gran cantidad de citoquinas (interferón gamma, factor de necrosis tumoralalfa, interleukina 1,6,12,18; factor estimulante de colonias de granulocitos/ macrófagos) induciendo hemofagocitosis ${ }^{4,5}$. Existe también apoptosis inadecuada de células inmunes ${ }^{10}$. Son características la hepatitis y colestasia secundarias a infiltración

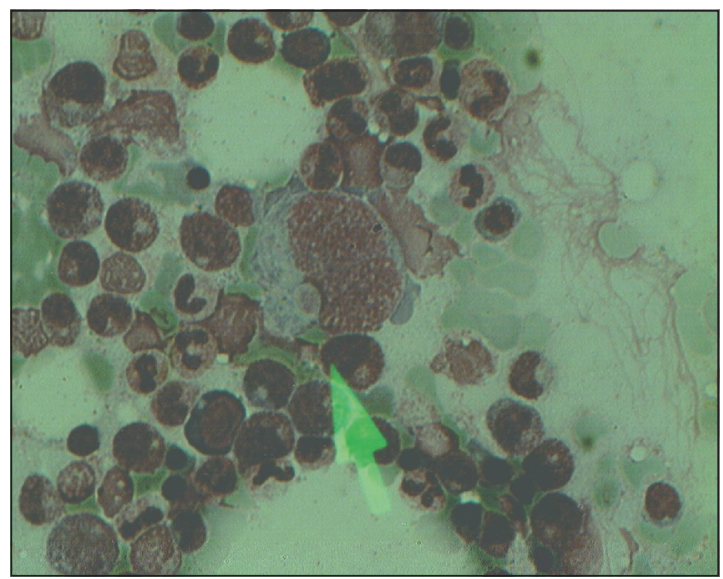

Figura 2. Frotis de médula ósea. Se observa un macrófago con un glóbulo rojo ya fagocitado en su citoplasma (flecha).

masiva de células T y macrófagos, aumento de la ferritina, hipertrigliceridemia, disminución del fibrinógeno y albúmina ${ }^{1,6,11}$. La hipertrigliceridemia se debe al aumento de VLDL por inhibición de lipasa lipoproteica por el factor de necrosis tumoral-alfa; la hiperferritinemia a la acumulación de ferritina en los macrófagos durante la eritrofagocitosis y al aumento de su síntesis mediada por interleukina 1; la hipofibrinogenemia por una liberación excesiva del factor activador del plasminógeno por macrófagos estimulados ${ }^{9,12}$. También pueden observarse hipoalbuminemia, ascitis, derrame pleural y elevación de la $\mathrm{LDH}^{11,13-16}$. Lo característico de este trastorno es la eritrofagocitosis medular, pero puede observarse en bazo, ganglios linfáticos, sistema nervioso central y en piel $^{4,5}$. Puede existir fagocitosis de leucocitos, linfocitos, plaquetas y precursores hematopoyéticos ${ }^{1}$. La hemofagocitosis también puede observarse como un fenómeno aislado en las anemias hemolíticas, enfermedades metabólicas y neoplasias?.

La anemia usualmente es grave, provocada por la eritrofagocitosis y el estado inflamatorio ${ }^{1}$. La hiperbilirrubinemia y elevación de la LDH pueden sugerir hemólisis pero el recuento de reticulocitos es bajo ${ }^{1}$. Marcadores inflamatorios como el receptor soluble de interleukina 2 (sCD25) o la ferritina pueden elevarse antes de que el SHF sea clínicamente aparente ${ }^{4}$.

Se han establecido criterios diagnósticos para el SHF, si bien pueden no presentarse todos inicialmente $^{4}$ : a) diagnóstico molecular de SHF o b) 5 de 
8 criterios: fiebre $\geq 38,5^{\circ} \mathrm{c}$, esplenomegalia, citopenias de al menos dos series, hipertrigliceridemia de ayuno y/o hipofibrinogenemia, hemofagocitosis, ausencia de actividad de células natural killer, ferritina $>500 \mathrm{ng} / \mathrm{mL}$ y aumento de sCD25.

Nuestro paciente cumplió 6 de esos criterios. Si bien el SHF se manifestó claramente después del rechazo agudo con la aparición de fiebre, pancitopenia e hiperferritinemia, es probable que las manifestaciones digestivas previas, la ascitis, derrame pleural, esplenomegalia e hipoalbuminemia hayan sido también secundarias al SHF, el cual estaba parcialmente controlado con el uso de ciclosporina (droga utilizada frecuentemente en su tratamiento). El estímulo inflamatorio originado en el rechazo agudo y la suspensión de CsA, pueden haber sido las causas de la exacerbación de un SHF larvado, ya presente. Hubo dos medidas que probablemente detuvieron el trastorno: la reinstauración de CsA y la suspensión definitiva del MMF, droga a la cual se ha atribuido el SHF ${ }^{16}$. La panhipogammaglobulinemia es explicada por la característica depresión de la actividad de los linfocitos B en el SHF ${ }^{17}$. No existen comunicaciones de la asociación entre síndrome de Alport y SHF.

El SHF se asocia a neoplasias malignas, infecciones, enfermedades colágeno-vasculares, inmunodeficiencias y trasplantes (Tabla 1).
Los trasplantados de órganos sólidos y hematopoyéticos pueden presentar un $\mathrm{SHF}^{1,6,7,13,14,18,19,21}$. En pacientes TxR la incidencia de SHF es $0,4 \%$ $0,5 \%^{7,13}$. La mayoría es gatillado por una infección viral, pero pueden desencadenarlo infecciones bacterianas, parasitarias, micóticas, tuberculosis y enfermedades linfoproliferativas ${ }^{1,7,22}$. La mayoría de los casos ocurre en las primeras semanas del TxR, después del día 10, cuando el paciente está intensamente inmunosuprimido o ha recibido terapia para un rechazo agudo ${ }^{1,7}$, como fue en este caso. Casi todos los pacientes experimentan fiebre y baja de peso. La mayoría presenta hepatomegalia y algunos adenopatías. La frecuencia de síntomas digestivos como diarrea, dolor abdominal, náuseas y vómitos la presenta más de $50 \%$ de los pacientes, las alteraciones hepáticas más de 50\%, la elevación de la ferritina $86 \%$, de triglicéridos $75 \%$ y de LDH $66 \%{ }^{7}$. Se recomienda que el tratamiento del SHF sea precoz dada la alta mortalidad ${ }^{4}$. En una recopilación de 34 casos sobrevivieron 15 y algunos perdieron el injerto ${ }^{7}$. El objetivo de la terapia es el control de la infección, la supresión de la hiperinflamación, la destrucción de las células infectadas presentadoras de antígenos y la atenuación de la función de los macrófagos activados ${ }^{22-24}$. Se ha empleado terapia anti-infecciosa empírica consistente en antibacterianos de amplio espectro,

\section{Tabla 1. Condiciones asociadas a síndrome hemofagocítico en adultos}

$\begin{array}{ll}\text { Neoplasias } & \text { Hematológicas: Linfomas no Hodgkin T, B y anaplásicos; mieloma múltiple, enfermedad de Hodgkin, } \\ & \text { leucemia mieloide aguda, leucemia linfática aguda de células T y B, eritroleucemia, linfomas y leuce- } \\ & \text { mias de células natural killer, granulomatosis linfomatoide } \\ & \text { No hematológicas: tumores mediastínicos de células germinales (no seminoma), sarcoma de Kaposi, } \\ & \text { meduloblastoma, sacrcoma de Ewing } \\ \text { Infecciones } & \text { Virales: Epstein Barr, citomegalovirus, influenza humana, porcina y aviar; herpes tipo 1, 2, 6, 8 y varice- } \\ & \text { la zoster; HIV, rubeola, parvovirus B19, virus A, C y E de hepatitis; adenovirus, enterovirus, hantavirus, } \\ & \text { dengue, coxsackie, sincicial respiratorio y BK } \\ & \text { Bacterianas: endocarditis infecciosa, sepsis, bartonellosis, fiebre Q, fiebre tifoídea, legionella, lep- } \\ & \text { tospiras, clamidias, brucelas, borrelia, Mycoplasma pneumoniae, micobacterias y enfermedad de } \\ & \text { Tsutsugamushi } \\ & \text { Micóticas: aspergillus, cándida, histoplasma, criptococo, tricospora y Pneumocistis jirovecci } \\ & \text { Parasitarias: leishmaniasis visceral, malaria, babesiosis y toxoplasmosis } \\ \text { Enfermedades } & \text { Lupus eritematoso sistémico, enfermedad de Still, esclerosis sistémica progresiva, artritis reumatoide, } \\ \text { colágeno-vasculares } & \text { artritis juvenil, enfermedad mixta del tejido conectivo, panarteritis nodosa, enfermedad de Kawasaki } \\ \text { Trasplantes e } & \text { Trasplantes: renal, hepático, riñón-páncreas, pulmón, hematopoyéticos } \\ \text { inmunodeficiencias } & \text { Inmunodeficiencias: congénitas y adquiridas, infección por VIH, anemia de células falciformes } \\ \text { Otras } & \text { Drogas: fenitoina, micofenolato, azatioprina, rituximab } \\ & \text { Idiopáticas, enfermedad de Kikuchi } \\ & \text { Formas familiares tardías }\end{array}$


antivirales, drogas antituberculosas y antifúngicas. Este tratamiento se ha modificado al identificar el patógeno responsable ${ }^{7}$. Si bien no existen estudios controlados, se han utilizado corticoides en dosis altas asociados a inmunosupresores ${ }^{6}$. Frecuentemente se ha usado la ciclosporina e inmunoglobulina endovenosa y con menor frecuencia tacrolimus ${ }^{1,7}$. Hay experiencias con etopósido, ciclofosfamida, globulina antitimocito, anticuerpos anti TNF, anti IL-1, anti IL-6, daclizumab y en ELPPT quimioterapia $(\mathrm{CHOP})$ y anticuerpos anti CD20 (rituximab) 2,4,6,7,9,22-24.

El SHF tiene mal pronóstico, a pesar del tratamiento ${ }^{7,22,15}$. La inflamación sostenida lleva a neutropenia y sepsis bacteriana o micótica. La causa más frecuente de fallecimiento es la falla orgánica múltiple (FOM $)^{6,9}$. En TxR es probable que algunas muertes en FOM sean secundarias a esta causa y que erróneamente hayan sido atribuidas a shock séptico ${ }^{14}$.

Este caso nos permite sugerir que frente a la brusca aparición de pancitopenia, fiebre y disfunciones orgánicas inexplicadas en un trasplantado, se considere al SHF en el diagnóstico diferencial. $\mathrm{Su}$ diagnóstico y tratamiento oportuno pueden evitar la muerte.

\section{Referencias}

1. Karras A. What nephrologists need to know about hemophagocytic syndrome. Nat Rev Nephrol 2009; 5: 329-36.

2. Coca A, Bundy KW, Marston B, Huggins J, Looney RJ. Macrophage activation syndrome: serological markers and treatment with anti-thymocyte globulin. Clin Immunol 2009; 132: 10-8.

3. Raschke RA, García-Orr R. Hemophagocytic lymphohistiocytosis: a potentially underrecognized association with systemic inflammatory response syndrome, severe sepsis, and septic shock in adults. Chest 2011; 140: 9338.

4. Jordan MB, Allen CE, Weitzman S, Filipovich AH, McClain KL. How I treat hemophagocytic lymphohistiocytosis. Blood 2011; 118: 4041-52.

5. Schwartz RA, Coppes MJ. Lymphohistiocytosis (Hemophagocytic Lymphohistiocytosis). http://emedicine. medscape.com/article/986458-overview Acceso el 21 de enero de 2011.

6. Shabbir M, Lucas J, Lazarchick J, Shirai K. Secondary hemophagocytic síndrome in adults: a case series of 18 patients in a single institution and review of literature. Hematol Oncol 2011; 29: 100-6.

7. Karras A, Thervet E, Legendre C, Groupe Coopératif de transplantation d'Ile de France. Hemophagocytic syndrome in renal transplant recipients: report of 17 cases and review of literature. Transplantation 2004; 77: 238-43.

8. Gauvin F, Toledano B, Champagne J, Lacroix J. Reactive hemophagocytic syndrome presenting as a component of multiple organ dysfunction syndrome. Crit Care Med 2000; 28: 3341-5.

9. Janka GE. Hemophagocytic syndromes. Blood Rev 2007; 21: 245-3.

10. Imashuku S, Ueda I, Teramura T, Mori K, Morimoto A, Sako M, et al. Occurrence of haemophagocytic lymphohistiocytosis at less than 1 year of age: analysis of 96 patients. Eur J Pediatr 2005; 164: 315-9.

11. Kim JM, Kwok SK, Ju JH, Kim HY, Park SH. Reactive hemophagocytic syndrome in adult korean patients with systemic lupus erythematosus: a case-control study and literature review. J Rheumatol 2012; 39: 86-93.

12. Verdugo LP, Rodríguez ZN, Tordecilla CJ, Soto AV. Síndrome hemofagocítico secundario en pediatría: Experiencia clínica en ocho casos. Rev Chil Pediatr 2005; $76: 397-403$.

13. Risdall RJ, McKenna RW, Nesbit ME, Krivit W, Balfour $\mathrm{HH}$ Jr, Simmons RJ, et al. Virus-associated hemophagocytic syndrome: a benign histiocytic proliferation distinct from malignant histiocytosis. Cancer 1979; 44: 993-1002.

14. Álvarez Sosa D, Pérez Tamajón L, Marrero Miranda D, González Rinne AM, Raya Sánchez JM, González-Posada Delgado JM. Linfohistiocitosis hemofagocítica: una rara y grave complicación en el trasplante renal. A propósito de tres casos. NefroPlus 2011; 4: 49-53.

15. Kürşat S, Cağirgan S, Ok E, Unsal A, Tokat $Y$, Saydam G, et al. Haemophagocytic-histiocytic syndrome in renal transplantation. Nephrol Dial Transplant 1997; 12: 105860.

16. Raffray L, Couzi L, Viallard JF, Pellegrin JL, Augis V, Vernant JP, et al. Mycophenolate mofetil: a possible cause of hemophagocytic syndrome following renal transplantation? Am J Transplant 2010; 10: 2378-9.

17. Ezdinli EZ, Kucuk O, Chedid A, Sinclair TF, Thomas K, Singh S, et al. Hypogammaglobulinemia and hemophagocytic syndrome associated with lymphoproliferative disorders. Cancer 1986; 57: 1024-37.

18. Díaz-Guzmán E, Dong B, Hobbs SB, Kesler MV, Hayes D Jr. Hemophagocytic lymphohistiocytosis after lung transplant: report of 2 cases and a literature review. Exp Clin Transplant 2011; 9: 217-22. 
19. George TI, Jeng M, Berquist W, Cherry AM, Link MP, Arber DA. Epstein-Barr virus-associated peripheral Tcell lymphoma and hemophagocytic syndrome arising after liver transplantation: case report and review of the literature. Pediatr Blood Cancer 2005; 44: 270-6.

20. Sreedharan A, Bowyer S, Wallace CA, Robertson MJ, Schmidt K, Woolfrey AE, et al. Macrophage activation syndrome and other systemic inflammatory conditions after BMT. Bone Marrow Transplant 2006; 37: 629-34.

21. Gurkan A, Yakupoglu U, Yavuz A, Dikici H, Yakupoglu YK, Tuncer $M$, et al. Hemophagocytic syndrome in kidney transplant recipients: report of four cases from a single center. Acta Haematol 2006; 116: 108-13.

22. Bea Granell S, Beneyto Castello I, Ramos Escorihuela D, Sánchez Plumed J, Sánchez Pérez P, Hernández-Jaras J, et al. Cytomegalovirus-associated haemophagocytic syndrome in a kidney transplant patient. Nefrologia 2011; 31: 236-8.

23. Janka G. Hemophagocytic lymphohistiocytosis: when the immune system runs amok. Klin Pediatr 2009; 221: 278-85.

24. Young P, Peroni J, Finn BC, Venditti JE, Preiti V, Bullorsky E, et al. [Hemophagocytic syndrome. Report of four cases]. Rev Med Chile 2011; 139: 224-9. 